\title{
On the Continued Fractions associated with the Hypergeometric Equation.
}

\author{
By E. Lindsay Ince, Carnegie Fellow.
}

(Received 29th January 1916. Read 11th February 1916).

\section{\$1. Introductory.}

The present paper is based on a method attributed to Euler of expressing as a continued fraction the logarithmic derivate of a solu tion of a linear differential equation of the second order. The method is particularly applicable to equations of hypergeometric type, and, in that connection, was previously employed by the present author * as a means of adding to the number of known transformations of continued fractions. The theory of the hypergeometric equation has been very thoroughly worked out, so that we may expect, by making use of the known properties of its solutions, to arrive at much that is new in the theory of continued fractions. Thus the investigation mentioned above was intended to show how to every transformation of one hypergeometric equation into another corresponds a transformation in continued fractions. The present paper makes use of the researches of Schwarz, in which he solved certain cases of the hypergeometric equation in a finite form, and indicates a method by which continued fractions may be derived which are expressible in an algebraic form.

§2. The two continued fractions derived from the hypergeometric equation.

Consider the hypergeometric equation

$$
x(1-x) \frac{d^{2} y}{d x^{2}}+\{\gamma-(\alpha+\beta+1) x\} \frac{d y}{d x}-\alpha \beta y=0 .
$$

Differentiating, it becomes

$$
x(1-x) \frac{d^{3} y}{d x^{3}}+\{\gamma+1-(\alpha+\beta+3) x\} \frac{d^{2} y}{d x^{2}}-(\alpha+1)(\beta+1) \frac{d y}{d x}=0,
$$

*. Proc. Edin. Math. Soc., Vol. XXXII. (1913-14), p. 101. 
and, in general, after differentiating $r$ times,

$$
\begin{aligned}
x(1-x) \frac{d^{r+2} y}{d x^{r+2}}+\{\gamma+r-(\alpha+\beta+2 r+1) x\} & \frac{d^{r+1} y}{d x^{r+1}} \\
& -(\alpha+r)(\beta+r) \frac{d^{r} y}{d x^{r}}=0 .
\end{aligned}
$$

From this set of recurrence-relations we are led at once to the continued fraction

$$
\begin{aligned}
\frac{1}{y} \frac{d y}{d x}=\frac{\alpha \beta}{\gamma-(\alpha+\beta+1) x}+ & \frac{(\alpha+1)(\beta+1) x(1-x)}{\gamma+1-(\alpha+\beta+3) x}+\ldots \\
& \frac{(\alpha+r)(\beta+r) x(1-x)}{\gamma+r-(\alpha+\beta+2 r+1) x}+\ldots
\end{aligned}
$$

Starting again from the hypergeometric equation (1), we may obtain a second set of recurrence-relations by repeated integration; thus, on integrating once, we obtain

$$
x(1-x) \frac{d y}{d x}+\{\gamma-1-(\alpha+\beta-1) x\} y-(\alpha-1)(\beta-1) y_{1}=0,
$$

and on integrating again,

$$
x(1-x) y+\{\gamma-2-(\alpha+\beta-3) x\} y_{1}-(\alpha-2)(\beta-2) y_{2}=0,
$$

and after integrating $r$ times,

$x(1-x) y_{r-2}+\{\gamma-r-(\alpha+\beta-2 r+1) x\} y_{r-1}-(\alpha-r)(\beta-r) y_{r}=0$

where $y_{1}$ denotes $\int y d x, y_{2}$ denotes $\int y_{1} d x$, and so on.

This set of recurrence-relations leads in its turn to the continued fraction

$$
\begin{aligned}
& \frac{1}{y} \frac{d y}{d x}=-\frac{\gamma-1-(\alpha+\beta-1) x}{x(1-x)}-\frac{(\alpha-1)(\beta-1)}{\gamma-2-(\alpha+\beta-3) x} \\
& -\frac{(\alpha-2)(\beta-2) x(1-x)}{\gamma-3-(\alpha+\beta-5) x}-\ldots-\frac{(\alpha-r)(\beta-r) x(1-x)}{\gamma-r-1-(\alpha+\beta-2 r-1) x-\ldots}
\end{aligned}
$$

These two continued fractions evidently converge most rapidly when the variable $x$ approaches the values 0 and 1 . In fact, when $\boldsymbol{x}$ approaches the value 0 , the continued fraction (A) tends towards the value $\frac{\alpha \beta}{\gamma}$, and when $x$ approaches the value 1 , it tends towards the value $\frac{\alpha \beta}{\gamma-\alpha-\beta-1}$ Likewise, when $x$ tends to zero, the continued fraction (B) approaches the value $\frac{1-\gamma}{x}$, and when $x$ tends to unity, it approaches the value $\frac{\gamma-\alpha-\beta}{x-1}$. 
Now, consider the typical solution of the differential equation (1) in the neighbourhood of its singular points $x=0$ and $x=1$. The exponents at the singularity $x=0$ are 0 and $1-\gamma$, and corresponding to these respectively

and

$$
y=1+\frac{\alpha \beta}{\gamma} x+\ldots, \text { whence } \frac{1}{y} \frac{d y}{d x}=\frac{\alpha \beta}{\gamma}+\ldots
$$

$$
\begin{gathered}
y=x^{1-\gamma}+\frac{(\alpha-\gamma+1)(\beta-\gamma+1)}{2-\gamma} x^{2-\gamma}+\ldots, \\
\text { whence } \frac{1}{y} \frac{d y}{d x}=\frac{1-\gamma}{x}+\frac{(\alpha-\gamma+1)(\beta-\gamma+1)}{2-\gamma}+\ldots
\end{gathered}
$$

Similarly, corresponding respectively to the exponents 0 and $\gamma-\alpha-\beta$ at the singularity $x=1$, we have $y=1+\frac{\alpha \beta}{\gamma-\alpha-\beta-1}(x-1)+\ldots$, whence $\frac{1}{y} \frac{d y}{d x}=\frac{\alpha \beta}{\gamma-\alpha-\beta-1}+\ldots$ and

$$
\begin{gathered}
y=(x-1)^{\gamma-\alpha-\beta}+\frac{(\gamma-\beta)(\gamma-\alpha)}{\alpha+\beta-\gamma-1}(x-1)^{\gamma-\alpha-\beta+1}+\ldots, \\
\text { whence } \frac{1}{y} \frac{d y}{d x}=\frac{\gamma-\alpha-\beta}{x-1}+\frac{(\gamma-\beta)(\gamma-\alpha)}{\alpha+\beta-\gamma-1}+\ldots
\end{gathered}
$$

all of which expansions proceed in ascending powers of $x$ or of $x-1$.

Comparing these expressions for $\frac{1}{y} \frac{d y}{d x}$ with the limiting values obtained directly from the two continued fractions, we arrive at the notable result that the continued fraction (A) corresponds to the expansions with zero exponent at each of the two singularities 0 and 1 , and the continued fraction $(\mathrm{B})$ to the expansions relative to the non-zero exponents. The continued fractions have, therefore, a range of convergence much more widely extended than any of the expansions in series. In fact, since the hypergeometric equation has no finite singularity other than $x=0$ and $x=1$, the continued fractions (A) and (B) would converge throughout the finite part of the $x$ plane, with the possible exception of the zeros of the series $y$.

But the two series $1+\frac{\alpha \beta}{\gamma} x+\ldots$ and $1+\frac{\alpha \beta}{\gamma-\alpha-\beta-1}(x-1)+\ldots$ are quite distinct and cannot be transformed into one another, so that we come to the conclusion that, even though the continued fraction (A) may converge throughout a region including the points $x=0$ and $x=1$, yet it represents in the neighbourhood of $x=0$ a function 
entirely different from that which it represents in the neighbourhood of $x=1$. Equations (4) and (5) of $\$ 4$ may be taken as examples of this phenomenon.

\section{\$3. The Solution in Finite Form.}

There are certain definite cases in which the hypergeometric equation admits of a solution in a finite form, and these cases are determined as follows :-

The Schwarzian derivative connected with the equation (1) is given by the relation

$$
\{s, x\}=\frac{1}{2} \frac{1-\lambda^{2}}{x^{2}}+\frac{1}{2} \frac{1-v^{2}}{(x-1)^{2}}+\frac{1}{2} \frac{\lambda^{2}-\mu^{2}+v^{2}-1}{x(x-1)}, \ldots \ldots
$$

where $\quad \lambda^{2}=(1-\gamma)^{2}, \quad \mu^{2}=(\alpha-\beta)^{2}, \quad \nu^{2}=(\gamma-\alpha-\beta)^{2}$.

Now there are fifteen separate cases, in which $\lambda, \mu, v$ are definite numerical constants, where a relation in finite terms exists between $x$ and $s$. When that is the case, the hypergeometric equation can be solved in a finite form. These fifteen cases may be tabulated as follows * :-

\begin{tabular}{llllll} 
Case & & & $\lambda$ & $\mu$ & $\nu$ \\
I. & $\ldots$ & $\ldots$ & $1 / 2$ & $1 / 2$ & $1 / n$ \\
II. & $\ldots$ & $\ldots$ & $1 / 2$ & $1 / 3$ & $1 / 3$ \\
III. & $\ldots$ & $\ldots$ & $2 / 3$ & $1 / 3$ & $1 / 3$ \\
IV. & $\ldots$ & $\ldots$ & $1 / 2$ & $1 / 3$ & $1 / 4$ \\
V. & $\ldots$ & $\ldots$ & $2 / 3$ & $1 / 4$ & $1 / 4$ \\
VI. & $\ldots$ & $\ldots$ & $1 / 2$ & $1 / 3$ & $1 / 5$ \\
VII. & $\ldots$ & $\ldots$ & $2 / 5$ & $1 / 3$ & $1 / 3$ \\
VIII. & $\ldots$ & $2 / 3$ & $1 / 5$ & $1 / 5$ \\
IX. $\quad \ldots$ & $\ldots$ & $1 / 2$ & $2 / 5$ & $1 / 5$ \\
X. & $\ldots$ & $\ldots$ & $3 / 5$ & $1 / 3$ & $1 / 5$ \\
XI. & $\ldots$ & $\ldots$ & $2 / 5$ & $2 / 5$ & $2 / 5$ \\
XII. & $\ldots$ & $\ldots$ & $2 / 3$ & $1 / 3$ & $1 / 5$ \\
XIII. & $\ldots$ & $4 / 5$ & $1 / 5$ & $1 / 5$ \\
XIV. $\ldots$ & $\ldots$ & $1 / 2$ & $2 / 5$ & $1 / 3$ \\
XV. & $\ldots$ & $\ldots$ & $3 / 5$ & $2 / 5$ & $1 / 3$ \\
\hline
\end{tabular}

"Schwarz, Crelle, lxxv. (1872), p. 282. 
Knowing the solution in any one of these cases, we may construct the relative continued fraction, and thus obtain an identity between a continued fraction and an algebraical expression.

Schematically represented, the hypergeometric equation is

$$
P\left\{\begin{array}{ccc}
0 & \infty & 1 \\
0 & \alpha & 0 \\
1-\gamma & \beta & \gamma-\alpha-\beta
\end{array}\right\}
$$

or, in terms of the new constants $\lambda, \mu, v$,

$$
P\left\{\begin{array}{cccc}
0 & \infty & 1 & \\
0 & \frac{1-\lambda-\mu-\nu}{2} & 0 & x \\
\lambda & \frac{1-\lambda+\mu-\nu}{2} & v &
\end{array}\right\}
$$

so that $\lambda, \mu, v$ are the exponent differences at the singularities $0, \infty, 1$ respectively, and may, without loss of generality, be taken to be always positive. As $\alpha$ and $\beta$ are interchangeable, we may further assume that $\alpha$ is greater than $\beta$, so that $\mu=\alpha-\beta$. We shall also limit ourselves to the case in which the ordinary hypergeometric series $F(\alpha, \beta, \gamma, x)$ converges right up to the point $x=1$, so that $\gamma-\alpha-\beta$ is positive and $\nu=\gamma-\alpha-\beta$. We then have $\lambda=1-\gamma$, or $\lambda=\gamma-1$, according as $\gamma$ is less or greater than unity. Thus, with these restrictions, we find that two distinct differential equations correspond to each set of values of $\lambda, \mu, v$, and as to each differential equation corresponds two distinct continued fractions we may ultimately arrive at 60 continued fraction relations of the kind required.*

\section{§4. A Case in Detail.}

Let us consider Case I. in which $\lambda=\frac{1}{2}, \mu=\frac{1}{2}, v=\frac{1}{n}$. On solving the relations $\lambda=1-\gamma, \mu=\alpha-\beta, \nu=\gamma-\alpha-\beta$, we find that

* Since the continued fractions in (A) and (B) are convergent at both the singular points 0 and 1 , we may discard the restriction that $\gamma-a-\beta>0$, and thus further increase the possible number of continued fraction relations. No further generality is, however, obtained by interchanging $\lambda$ and $\nu$. 
$\gamma=\frac{1}{2}, \quad \alpha=\frac{1}{2}-\frac{1}{2 n}, \quad \beta=-\frac{1}{2 n}$, and the corresponding hypergeometric equation is

$$
\begin{gathered}
x(1-x) \frac{d^{2} y}{d x^{2}}+\left\{\frac{1}{2}-x\left(\frac{3}{2}-\frac{1}{n}\right)\right\} \frac{d y}{d x}+\frac{1}{4 n}\left(1-\frac{1}{n}\right) y=0 \ldots \\
\quad \text { or } \quad P\left\{\begin{array}{cccc}
0 & \infty & 1 \\
0 & \frac{1}{2}-\frac{1}{2 n} & 0 & x \\
\frac{1}{2} & -\frac{1}{2 n} & \frac{1}{n}
\end{array}\right\}
\end{gathered}
$$

The quotient, $s$, of the two solutions of this equation is given by

so that

$$
s=\frac{(1+\sqrt{x})^{2 / n}}{(1-x)^{1 / n}}
$$

$$
s^{\prime}=\frac{d s}{d x}=\frac{1}{n} x^{-1 / 2}(1+\sqrt{x})^{2 / n}(1-x)^{-1-1 / n}
$$

The complete primitive of (3) then is

$$
\begin{aligned}
y & =\left(A s^{\prime-1 / 2}+B s^{\prime-1 / 2} s\right) x^{-1 / 4}(1-x)^{-1 / 2-1 / 2 n} \\
& =A^{\prime}(1-x)^{1 / n}(1+\sqrt{x})^{-1 / n}+B^{\prime}(1+\sqrt{x})^{1 / n} \\
& =A^{\prime}(1-\sqrt{x})^{1 / n}+B^{\prime}(1+\sqrt{x})^{1 / n}
\end{aligned}
$$

where $A, B, A^{\prime}, B^{\prime}$ are arbitrary constants.

Consider the behaviour near the singularity $x=1$ of the two parts of this primitive. Writing $1-x=\xi$ it becomes

$$
A^{\prime} \xi^{1 / n}\{1+\sqrt{1-\xi}\}^{-1 / n}+B^{\prime}\{1+\sqrt{1-\xi}\}^{1 / n}
$$

Near $\xi=0$, i.e. near $x=1,\{1+\sqrt{1-\xi}\}^{1 / n}$ can be expended as a Taylor's series in powers of $\xi$, so that this particular solution is the one which corresponds to the exponent 0 at the singularity $x=1$ of the differential equation The quantity $\{1+\sqrt{1-\xi}\}^{-1 / n}$ can likewise be expanded near $\xi=0$ as a Taylor's series in powers of $\xi$, but the particular solution into which it enters contains also the multiplicative factor $\xi^{1 / n}$, and therefore corresponds to the exponent $\frac{1}{n}$ at the singularity $x=1$ of the differential equation. Thus in the neighbourhood of the singularity $x=1$ the solution 
$(1+\sqrt{x})^{1 / n}$ corresponds to the continued fraction which we have called $(A)$, and the solution $(1-x)^{1 / n}(1+\sqrt{x})^{-1 / n}$ to the continued fraction (B).

It is, however, easily seen that, as might be expected, the solution $(1+\sqrt{x})^{1 / n}$ does not also correspond to the exponent 0 at the singularity $x=0$, nor does the solution $(1-\sqrt{x})^{1 / n}$ correspond to the non-zero exponent at that singularity. In fact, it may readily be verified that the appropriate solutions at the singularity $x=0$ are respectively

$$
\frac{1}{2}\left\{(1+\sqrt{x})^{1 / n}+(1-\sqrt{x})^{1 / n}\right\} \text { and } \frac{n}{2}\left\{(1+\sqrt{x})^{1 / n}-(1-\sqrt{x})^{1 / n}\right\} .
$$

We may thus deduce the two continued-fraction relations :-

$$
\begin{gathered}
\frac{1-\frac{1}{n}}{1-\left(3-\frac{2}{n}\right) x}+\frac{\left(2-\frac{1}{n}\right)\left(3-\frac{1}{n}\right) x(1-x)}{3-\left(7-\frac{2}{n}\right) x+\frac{\left(4-\frac{1}{n}\right)\left(5-\frac{1}{n}\right) x(1-x)}{5-\left(11-\frac{2}{n}\right) x}+\ldots}+\frac{\left(2 r-\frac{1}{n}\right)\left(2 r+1-\frac{1}{n}\right) x(1-x)}{2 r+1-\left(4 r+3-\frac{2}{n}\right) x}+\ldots \\
\ldots+\frac{\left\{(1+\sqrt{ } x)^{1 / n-1}-(1-\sqrt{ } x)^{1 / n-1}\right\}}{\left\{(1+\sqrt{ } x)^{1 / n}+(1-\sqrt{ } x)^{1 / n}\right\} \sqrt{ } x} \text { in the neighbourhood of } x=0 \\
=-x^{-1 / 2}(1+\sqrt{ } x)^{-1} \text { in the neighbourhood of } x=1 \text {............. (4) }
\end{gathered}
$$

and

$$
\begin{gathered}
\frac{\left(1+\frac{1}{n}\right)\left(2+\frac{1}{n}\right)}{-3+\left(5+\frac{2}{n}\right) x}-\frac{\left(3+\frac{1}{n}\right)\left(4+\frac{1}{n}\right) x(1-x)}{-5+\left(9+\frac{2}{n}\right) x-\ldots-\frac{\left(2 r-1+\frac{1}{n}\right)\left(2 r+\frac{1}{n}\right) x(1-x)}{-2 r-1+\left(4 r+1+\frac{2}{n}\right) x-. .}} \\
=\frac{1-\left(1+\frac{2}{n}\right) x}{x(1-x)}-\frac{\left\{(1+\sqrt{x})^{1 / n-1}+(1-\sqrt{ } x)^{1 / n-1}\right\}}{n\left\{(1+\sqrt{x})^{1 / n}-(1-\sqrt{ } x)^{1 / n}\right\} \sqrt{ } x} \\
\text { in the neighbourhood of } x=0 \\
=\frac{1}{x}+\frac{1}{n} x^{-1 / 2}(1+\sqrt{x})^{-1} \text { in the neighbourhood of } x=1 . . .(5)
\end{gathered}
$$


The second differential equation

$x(1-x) \frac{d^{2} y}{d x^{2}}+\left\{\frac{3}{2}-\left(\frac{5}{2}-\frac{1}{n}\right) x\right\} \frac{d y}{d x}-\left(1-\frac{1}{2 n}\right)\left(\frac{1}{2}-\frac{1}{2 n}\right) y=0$

of Case I. may be similarly treated, and will be found to give rise to the same two continued fractions as occur in (4) and (5), though in the reverse order. In like manner the remaining fourteen cases may be worked out, the differential equations of each case leading to a pair of continued fraction relations.

\section{§5. The cases of $\lambda+\mu+\nu=1$.}

The cases enumerated by Schwarz are characterised by the inequality $\lambda+\mu+v>1$. When $\lambda+\mu+\nu=1$, there is a simple method by which algebraic solutions of the hypergeometric equation may be obtained. Forsyth (Differential Equations, 4th Edition, Ex. 3, p. 121) gives the following examples, which may conceivably give rise to new continued fraction relations, viz.
Case (i) $\beta=1$

$$
\lambda^{2}=(1-\gamma)^{2}
$$$$
\mu^{2}=(\alpha-1)^{2}
$$$$
v^{2}=(\gamma-\alpha-1)
$$
(ii) $\gamma=\alpha$
$\lambda^{2}=(1-\alpha)^{2}$
$\mu^{2}=(\alpha-\beta)^{2} \quad \nu^{2}=\beta^{2}$
(iii) $\gamma=\alpha+1 \quad \lambda^{2}=\alpha^{2}$
$\mu^{2}=(\alpha-\beta)^{2} \quad \nu^{2}=(1-\beta)^{2}$.

Cases (ii) and (iii) are virtually the same, and do not lead to distinct continued fractions. Taking case (ii) we find that a solution of the corresponding differential equation

$$
x(1-x) \frac{d^{2} y}{d x^{3}}+\{\alpha-(\alpha+\beta+1) x\} \frac{d y}{d x}-\alpha \beta y=0
$$

is $(x-1)^{-\beta}$,

which represents the solution corresponding to the exponent $-\beta$ at the singularity $x=1$ and the exponent 0 at the singularity $x=0$. Thus we are led to the two continued fraction relations

$$
\begin{array}{r}
\frac{\alpha \beta}{\alpha-(\alpha+\beta+1) x}+\frac{(\alpha+1)(\beta+1) x(1-x)}{\alpha+1-(\alpha+\beta+3) x}+\ldots \\
\ldots+\frac{(\alpha+r)(\beta+r) x(1-x)}{\alpha+r-(\alpha+\beta+2 r+1) x}+\ldots \\
\quad=\frac{\beta}{1-x} \text { in the neighbourhood of } x=0 \ldots \ldots
\end{array}
$$




\section{4}

and

$$
\begin{aligned}
\frac{(\alpha-1)(\beta-1)}{\alpha-2-(\alpha+\beta-3) x}+ & \frac{(\alpha-2)(\beta-2) x(x-1)}{\alpha-3-(\alpha+\beta-5) x}+\ldots \\
& \frac{(\alpha-r)(\beta-r) x(x-1)}{\alpha-r-1-(\alpha+\beta-2 r-1) x+\ldots} \\
= & \frac{1-\alpha}{x} \text { in the neigbourhood of } x=1 . \ldots . .
\end{aligned}
$$

These two continued fractions may easily be transformed into one another. The second solution has not in general an algebraic form. In case (i) the continued fraction of (B) vanishes, and we are led to a simple identity, while the continued fraction (A) is not expressible algebraically. 